\title{
Crystallographic fragment screening of SARS-CoV-2 drug discovery targets
}

\section{Daren Fearon ${ }^{1,2}$, Alice Douangamath ${ }^{1,2}$, Ailsa Powell ${ }^{1,2}$, Alex Dias ${ }^{1,2}$, Anthony Aimon ${ }^{1,2}$, Jose Brandao-Neto ${ }^{1,2}$, Louise Dunnett $^{1,2}$, Rachael Skyner ${ }^{1,2}$, Tyler Gorrie-Stone ${ }^{1,2}$, Warren Thompson ${ }^{1,2}$, Frank von Delft ${ }^{1,2,3,4}$}

${ }^{1}$ Diamond Light Source Ltd., Harwell Science and Innovation Campus, Didcot, OX11 0QX, UK; ${ }^{2}$ Research Complex at Harwell, Harwell Science and Innovation Campus, Didcot, OX11 OFA, UK; ${ }^{3}$ Centre for Medicines Discovery, University of Oxford, Old Road

Campus, Roosevelt Drive, Headington, OX3 7DQ, UK; ${ }^{4}$ Department of Biochemistry, University of Johannesburg, South Africa

\section{daren.fearon@diamond.ac.uk}

SARS-CoV-2 is a novel coronavirus and causative agent of the zoonotic disease Covid-19, which has been responsible for over 3 million deaths globally. Although the rapid development of several highly efficacious vaccines is proving effective in reducing the spread and severity of the disease, the development of novel, low cost and globally available anti-viral therapeutics remains an essential goal, both for this pandemic and for future outbreaks of related coronaviruses.

To identify starting points for such therapeutics, the XChem team at Diamond Light Source, in collaboration with various international colleagues, have performed large crystallographic fragment screens against 7 key SARS-CoV-2 proteins including the Main protease, the Nsp3 macrodomain and the helicase Nsp13 [1-3]. The expeditious collection and dissemination of data from these screens has been enabled by the well-established platform at Diamond and by the implementation of various new tools in the XChem pipeline.

This work has identified numerous starting points for the development of more potent inhibitors as exemplified by the ongoing work from the open science drug discovery project, the Covid Moonshot [4]. By merging fragment hits from the initial XChem screen and harnessing crowdsourced medicinal chemistry designs from the global community we have been able to rapidly develop potent inhibitors of the Main protease that exhibit promising antiviral activity.

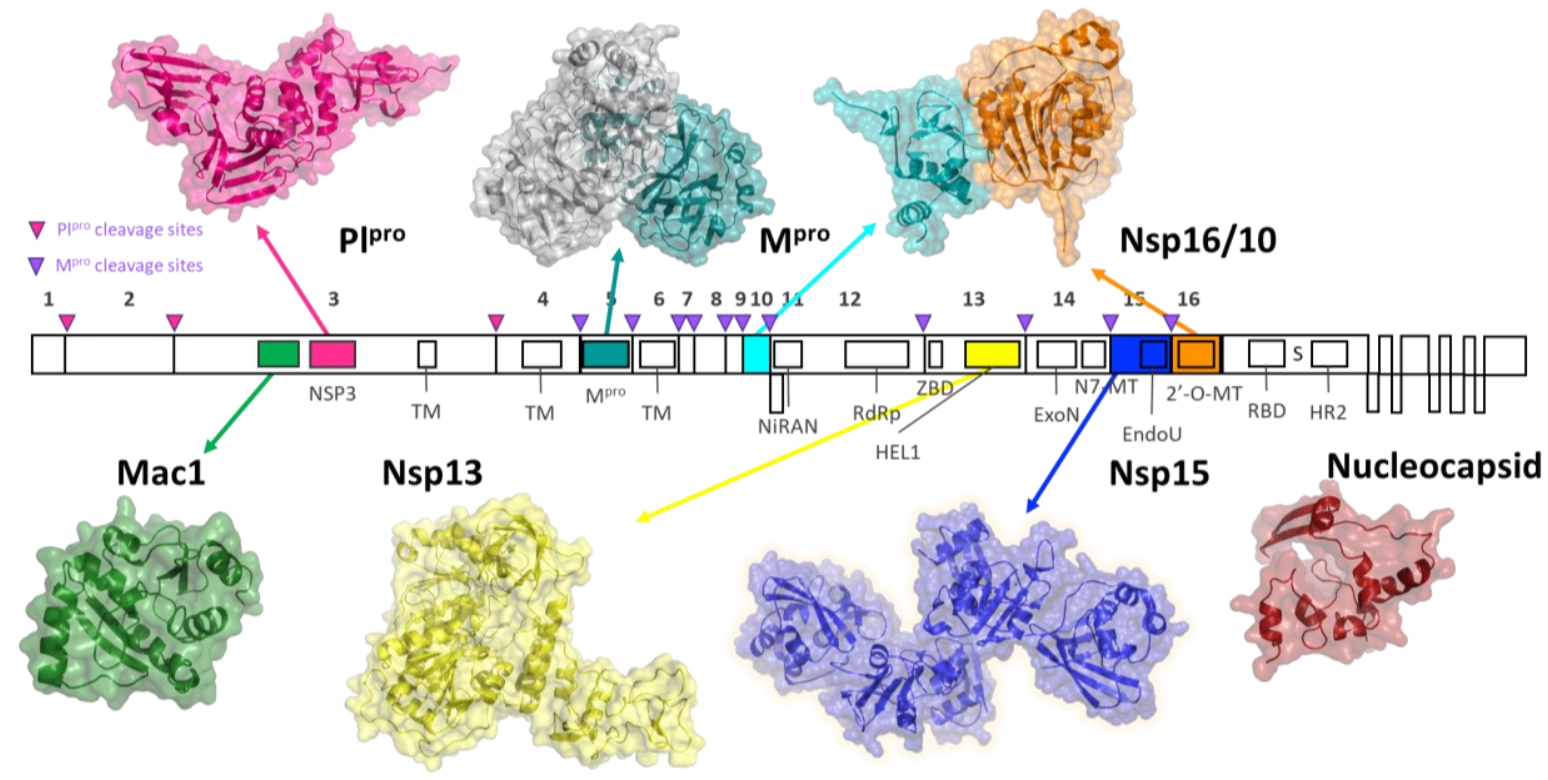

Figure 1. SARS-CoV-2 genome and proteins screened on the XChem platform at Diamond Light Source

[1] Douangamath, A., et al., Nature Communications, 11, 2020.

[2] Schuller, M., et al., Science Advances, 7, 2021.

[3] Newman, J., et al., BioRxiv, 2021.

[4] The COVID Moonshot Consortium, BioRxiv, 2021.

Keywords: XChem; coronavirus; fragments; FBDD

COVID Moonshot Consortium

Acta Cryst. (2021), A77, C196 